\title{
Quadrate Lobe of Liver
}

National Cancer Institute

\section{Source}

National Cancer Institute. Quadrate Lobe of Liver. NCI Thesaurus. Code C112404.

An oblong shaped area of the liver that is situated inferior to the right lobe, bounded by the anterior marg in of the liver and the porta hepatis. 